\title{
In-situ TEM Study of Sintering of Capped Silver Nanoparticles
}

\author{
M.A. Asoro*, D. Kovar*†, P.J. Ferreira*† \\ *Materials Science and Engineering Program and $†$ Department of Mechanical Engineering, \\ University of Texas at Austin, Austin, TX 78712
}

Nanoparticles possess unique properties that are different from that of bulk material, providing new applications in many areas of science and technology. For example, nanoparticles are currently used as catalysts for chemical reactions and for patterned metallic conductors in microelectronics. However, due to their large surface area to volume ratio, nanoparticles have a strong tendency to agglomerate and sinter during processing or usage over short time scales and at temperatures lower than bulk sintering temperatures. This in turn leads to a change in their properties.

In this work, we investigate how particle size, temperature and nanoparticle capping layer influence sintering of silver nanoparticles using a transmission electron microscope (TEM) equipped with a novel Protochips Aduro ${ }^{\mathrm{TM}}$ heating stage to carry out in-situ heating experiments. This heating stage enables very fast heating rates $\left(10^{6}{ }^{\circ} \mathrm{C} / \mathrm{s}\right)$ with an extremely low thermal drift even at high temperatures due to its low mass [1]. We use commercially available silver nanoparticles (from Nanotechnologies Inc.) that typically have a surfactant or organic capping layer on the surface to prevent agglomeration of the nanoparticles.

A sequence of in-situ heating TEM images showing sintering of two $15 \mathrm{~nm}$ silver nanoparticles at $200{ }^{\circ} \mathrm{C}$ is shown in Fig. 1. From these in-situ TEM images, the particle radius, neck radius and dihedral angle were measured (Fig. 2) using a procedure that has been described previously [2]. These values were subsequently used to calculate the surface diffusion coefficient. For surface diffusion, the change in neck radius $(x)$ with time $(t)$ for a given particle radius $(r)$ and temperature $(T)$ is given by the following expression $[3,4]$

$$
x^{7}=\frac{56 \Omega r^{3} \gamma_{s} D_{s} \delta_{s} t}{K T}
$$

where $\Omega=$ atomic volume, $\gamma_{s}=$ surface energy, $D_{s}=$ surface diffusivity, $\delta_{s}=$ surface diffusive width and $K=$ Boltzmann's constant. Also, an in-situ TEM heating experiment was performed on two $40 \mathrm{~nm}$ silver nanoparticles at $400{ }^{\circ} \mathrm{C}$ and a similar analysis was done to determine the sintering parameters. From these experiments, the surface diffusion coefficient, $D_{s}$ of silver nanoparticles with an organic capping layer was calculated to be in the range $1.04-1.55 \times 10^{-21}$ $\mathrm{m}^{2} / \mathrm{s}$ at $200{ }^{\circ} \mathrm{C}$ and $2.76-5.11 \times 10^{-20} \mathrm{~m}^{2} / \mathrm{s}$ at $400{ }^{\circ} \mathrm{C}$. These values of surface diffusivity when compared with data obtained from bulk material at high temperatures and extrapolated to lower temperatures are significantly lower than the bulk silver estimates. This is believed to be due to the surfactant or capping layer on the nanoparticles that retards the sintering of the nanoparticles. To test this hypothesis, this data will be compared in the future to similar experiments conducted on silver nanoparticles that are free of capping layers. 


\section{References}

[1] L. F. Allard, W. C. Bigelow, M. Jose-Yacaman, D. P. Nackashi, J. Damiano, S. E. Mick, Microscopy Research and Technique 72 (2009) 208-215.

[2] M. A. Asoro, D. Kovar, Y. Shao-Horn, L. F. Allard, P. J. Ferreira, Nanotechnology 21 (2010) 025701.

[3] W. D. Kingery, M. Berg, Journal of Applied Physics 26 (1955) 1205-1212.

[4] G. C. Kuczynski, Trans. AIME 185 (1949) 169-178.
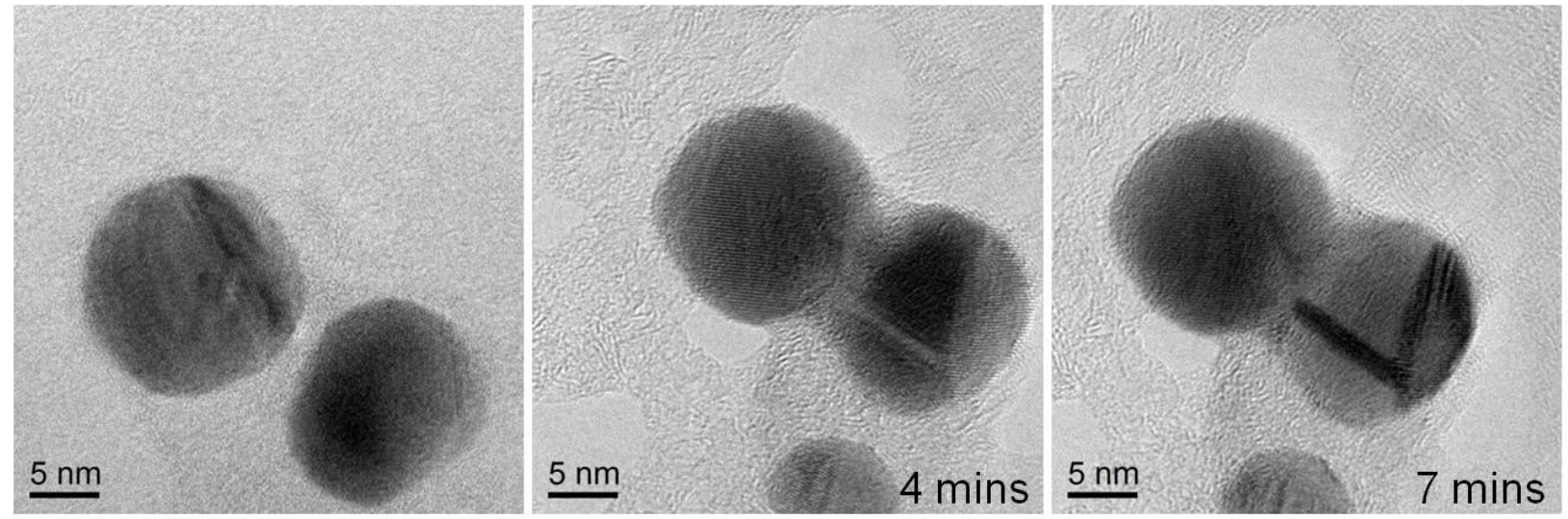

Figure 1: A sequence of in-situ TEM heating images showing sintering of silver nanoparticles at (a) RT (b) $200{ }^{\circ} \mathrm{C}$ after 4 minutes (c) $200{ }^{\circ} \mathrm{C}$ after 7 minutes
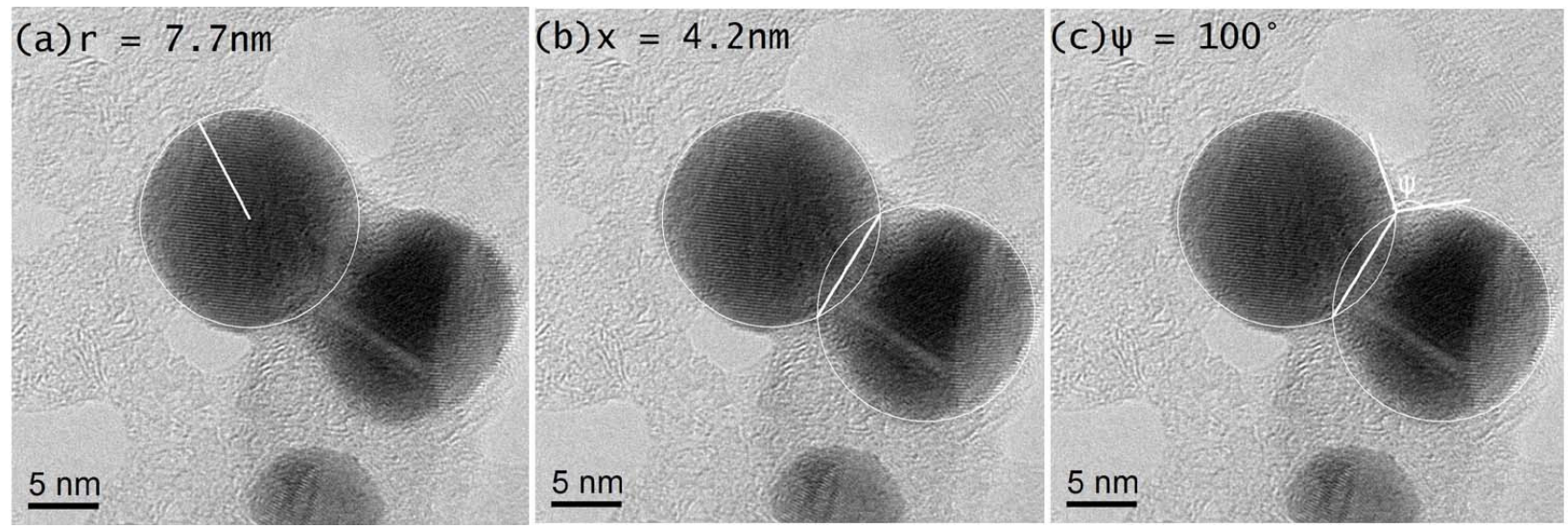

Figure 2: In-situ heating TEM images of silver nanoparticles from Fig. $1 \mathrm{~b}$ showing the measurements of (a) particle radius (b) neck radius (c) dihedral angle. 